\title{
SURFACE STATE OF Gd(0001) FILMS ON W(110): SCANNING TUNNELING SPECTROSCOPY STUDY
}

\author{
M. Bode, R. Pascal, M. Getzlaff and R. Wiesendanger \\ Institute of Applied Physics and Microstructure Research Center \\ University of Hamburg, Jungiusstrasse 11, 20355 Hamburg, Germany
}

\begin{abstract}
The surface electronic structure of $\mathrm{Gd}(0001) / \mathrm{W}(110)$ has been studied by scanning tunneling spectroscopy in dependence of the coverage. An exchange split $d_{z^{2}}$-like surface state which is well known from photoemission experiments was already found for coverages as low as $2 \mathrm{ML}$. The exchange splitting was found to increase with increasing coverage up to the fourth ML. For higher coverages the electronic structure remains constant. We found spectra that are shifted by $\approx 25 \mathrm{meV}$, possibly due to stacking faults.
\end{abstract}

PACS numbers: 73.20.At, 68.55.Jk, 61.16.Ch

\section{Introduction}

Heteroepitaxially grown thin films may exhibit physical properties which do not exist in nature due to their reduced symmetry, interface effects, and non-natural crystallographic structure. Therefore the design of layered materials enables one to perform real material engineering and causes the substantial scientific and technological interest into heteroepitaxial film growth especially in the data storage business. In the past the electronic properties of thin films and multilayers have usually been studied by spatially averaging techniques like photoemission spectroscopy (PES) and inverse photoemission spectroscopy (IPES) which cause severe problems to interpret data obtained from samples with complex morphologies. It is a non-trivial task to attribute certain signatures in the spectra to different species and crystallographic structures which may be present on the sample surface. Since the invention of the scanning tunneling microscope [1] and its spectroscopical measurement techniques $[2,3]$ it is possible to measure electronic properties like the density of state or the barrier height [4] spatially resolved in real space down to the atomic scale.

In the past we have applied scanning tunneling spectroscopy (STS) to investigate the electronic structure of thin Fe- and submonolayer Gd-films on W(110) [5-7]. Here we report on spectroscopy of the rare-earth (RE) metal gadolinium for coverages above one monolayer (ML). The (0001) surface of Gd exhibits a $d_{z^{2}}$-like 
surface state $[8,9]$ which was made responsible for extraordinary surface magnetic properties [10,11], e.g. an enhanced surface Curie-temperature [12]. Previous studies revealed that the bulk Curie-temperature of $\mathrm{Gd}(0001)$ films decreases with decreasing coverage [13]. However, little is known about the dependence of the surface Curie-temperature on the Gd film thickness. Since the RE(0001) surface state seems to be closely connected with the existence of an enhanced surface Curie-temperature as mentioned above, we decided to study the surface electronic properties of $\operatorname{Gd}(0001)$ in dependence of the coverage. We will show that the surface state exists and is exchange split for local coverages $\Theta_{\text {loc }} \geq 2$ ML. The exchange splitting increases up to the 4th $\mathrm{ML}$ and remains constant for higher coverages up to approximately $30 \mathrm{ML}$. Homoepitaxial growth of Gd/Gd(0001) results in triangular shaped islands. Above some of these islands we measured peaks in the differential conductivity $\mathrm{d} I / \mathrm{d} U$ which are shifted by $\approx 25 \mathrm{mV}$. One possible explanation are stacking-faults between the surface and the first subsurface layer of $\mathrm{Gd}(0001)$.

\section{Experimental}

The experiments have been performed in a UHV-system with different chambers for substrate preparation, film deposition, surface analysis, and sample transfer. The base pressure was always below $p=1 \times 10^{-10} \mathrm{mbar}$. We used W(110)-substrates which were cleaned by cycles of heating in an oxygen atmosphere $\left(p_{\mathrm{O}_{2}} \approx\right.$ $5 \times 10^{-7} \mathrm{mbar}$ ) and flashing up to $2500 \mathrm{~K}$ [14]. Gd was deposited by means of electron bombardment evaporation. During evaporation the pressure did not exceed $p=4 \times 10^{-10} \mathrm{mbar}$. A sharp hexagonal LEED-pattern and an intensive $d_{z^{2}}$-like surface state measured by means of photoemission spectroscopy (PES) were taken as indicators for well-ordered and clean $\mathrm{Gd}(0001)$-films.

We used a commercial variable-temperature scanning tunneling microscope (VT-STM) in this study. The sample could be cooled as low as $20 \mathrm{~K}$. Since the sample is rigidly clamped into a copper block we estimate that the overall accuracy of the temperature measurement is $\pm 5 \mathrm{~K}$. All topographic data were achieved in the constant current mode. To measure the $\mathrm{d} I / \mathrm{d} U$-signal by lock-in technique an ac-component $\left(U_{\mathrm{mod}} \leq 20 \mathrm{mV}, \nu \approx 325 \mathrm{~Hz}\right.$ ) was added to the gap voltage $U$. Since the tunneling current $I$ was measured simultaneously we are able to fit the lock-in signal with the differentiated $I$ vs. $U$ spectrum and therefore to quantify the differential conductivity in $\mathrm{A}$ per $\mathrm{V}$ instead of arbitrary units as usually done. In the following we will refer the bias voltages to the sample. Therefore the electrons tunnel from the tip towards the sample at positive bias (detection of empty sample states) and vice versa (detection of occupied sample states).

\section{Results and discussion}

Figure la shows the topography of approximately $5 \mathrm{ML}$ Gd evaporated on the W(110) substrate held at $530 \mathrm{~K}$. As reported by Aspelmeier et al. in Ref. [15] this results in the formation of islands instead of a smooth film. Since the Gd islands are atomically flat and the substrate exhibits several one-atomic steps below the island surface, the local coverage $\Theta_{\text {loc }}$ decreases for every island from the left towards the right edge from maximal $22 \mathrm{ML}$ down to $4 \mathrm{ML}$. Between the islands 

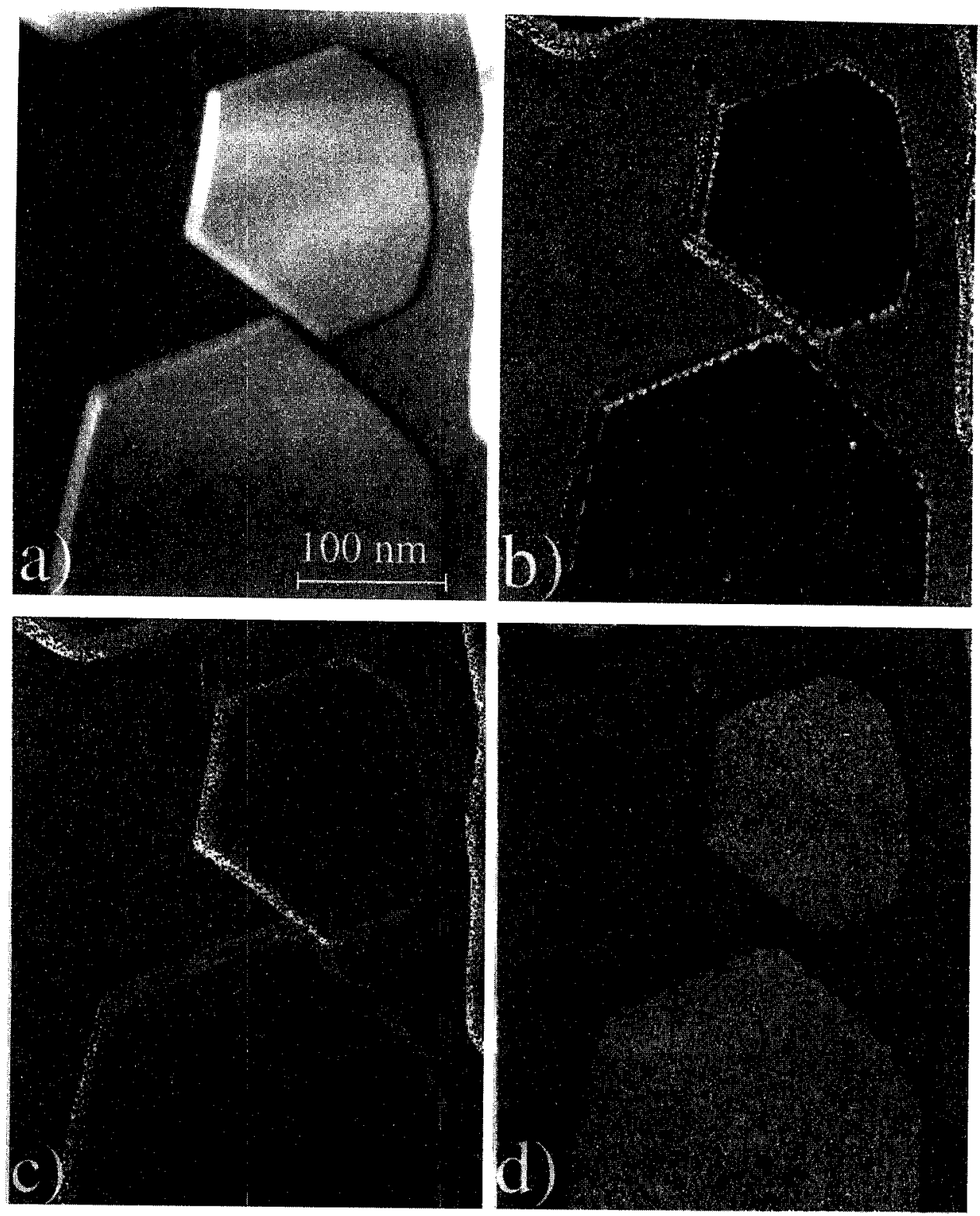

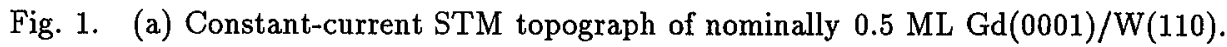
Since the substrate was held at $530 \mathrm{~K}$ during evaporation this results in StranskiKrastanov-growth. The substrate exhibits steps below the atomically flat islands. Therefore, the local Gd coverage decreases from the left edge of the islands to the right edge. Maps of the differential conductivity $\mathrm{d} I / \mathrm{d} U$ measured at different sample bias are shown in (b) $U=+0.8 \mathrm{~V}$, (c) $U=+0.47 \mathrm{~V}$, and (d) $U=-0.1 \mathrm{~V}$. The $\mathrm{d} I / \mathrm{d} U$-signal and therefore the local density of states does not depend on the local coverage. 
the substrate is covered with a stressed hexagonal monolayer of Gd which has been described already in Refs. [16] and [17]. Simultaneously with the topography we have measured the $\mathrm{d} I / \mathrm{d} U$-spectrum at every pixel of the scan. During the measurement the sample was held at $T=293 \mathrm{~K}$. Figures $1 \mathrm{~b}-\mathrm{d}$ show maps of the differential conductivity $\mathrm{d} I / \mathrm{d} U$ for different sample bias: (b) $U=+0.8 \mathrm{~V}$, (c) $U=+0.47 \mathrm{~V}$, and (d) $U=-0.1 \mathrm{~V}$. The differential conductivity is greycoded, i.e. the higher the local $\mathrm{d} I / \mathrm{d} U$-signal the brighter a location appears. At a sample bias $U=+0.8 \mathrm{~V}$ the tunneling current is dominated by electrons which tunnel from the tip into unoccupied sample states with a binding energy of $+0.8 \mathrm{eV}$. Comparison with the topographic data of Fig. 1a reveals that at this particular binding energy the differential conductivity above the Gd monolayer is higher than above any island. Beside few small bright spots which represent a change of the local electronic properties induced by the local adsorption of hydrogen and which has been described elsewhere [18] the $\mathrm{d} I / \mathrm{d} U$-signal at $U=+0.8 \mathrm{~V}$ measured above the $\mathrm{Gd}$ island is uniform and therefore independent on the local coverage in the range $22 \leq \Theta_{\text {loc }} \leq 4$. The total amount of hydrogen adsorbed on the surface is far less than $0.01 \mathrm{~L}$ ( 1 langmuir $=1 \times 10^{-6}$ torr $\left.\cdot \mathrm{s}\right)$. In the $\mathrm{d} I / \mathrm{d} U$-maps we never found a contrast on the Gd island at aniy sample bias (cf. Figs. 1b-d) in the voltage range under study $(-0.6 \mathrm{~V} \leq U \leq+0.9 \mathrm{~V})$. At $U=+0.47 \mathrm{~V}$ the contrast between the island surface and the monolayer vanishes. The contrast inverts if the sample bias is further reduced. For instance Fig. 1d shows a map of the $\mathrm{d} I / \mathrm{d} U$-signal at $U=-0.1 \mathrm{~V}$.

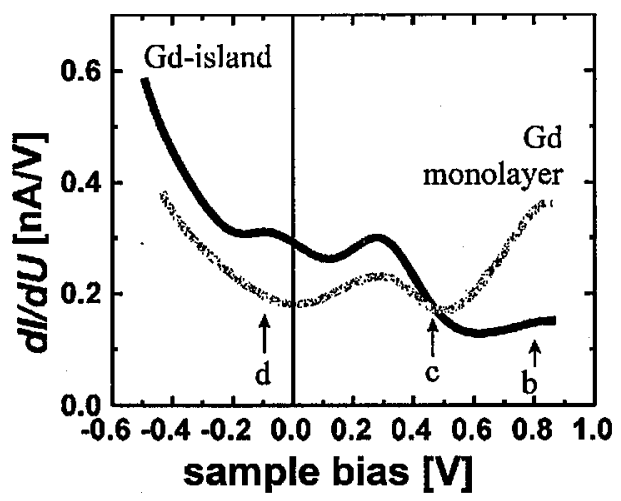

Fig. 2. Tunneling spectra measured above the Gd(0001) island (black) and above the first stressed hexagonal monolayer $\mathrm{Gd} / \mathrm{W}(110)$ (grey). The arrows $b-d$ indicate the bias voltage at which the $\mathrm{d} I / \mathrm{d} U$-maps as shown in Figs. $1 \mathrm{~b}-\mathrm{d}$ have been measured.

This behavior can be understood by comparing averaged tunneling spectra measured above Gd islands and above the Gd monolayer (Fig. 2). At high positive sample bias the $\mathrm{d} I / \mathrm{d} U$-signal and therefore the local density of states (LDOS) above the Gd monolayer is higher than above the island. The difference decreases with decreasing sample bias and vanishes at $U=0.47 \mathrm{~V}$ (cf. Fig. 1c). Both, the monolayer and the island spectrum exhibit a peak in the $\mathrm{d} I / \mathrm{d} U$-signal at positive sample bias $(U=+0.3 \mathrm{~V})$. However, the peak as measured above the island is more intensive. A weak peak can be recognized in the island spectrum 


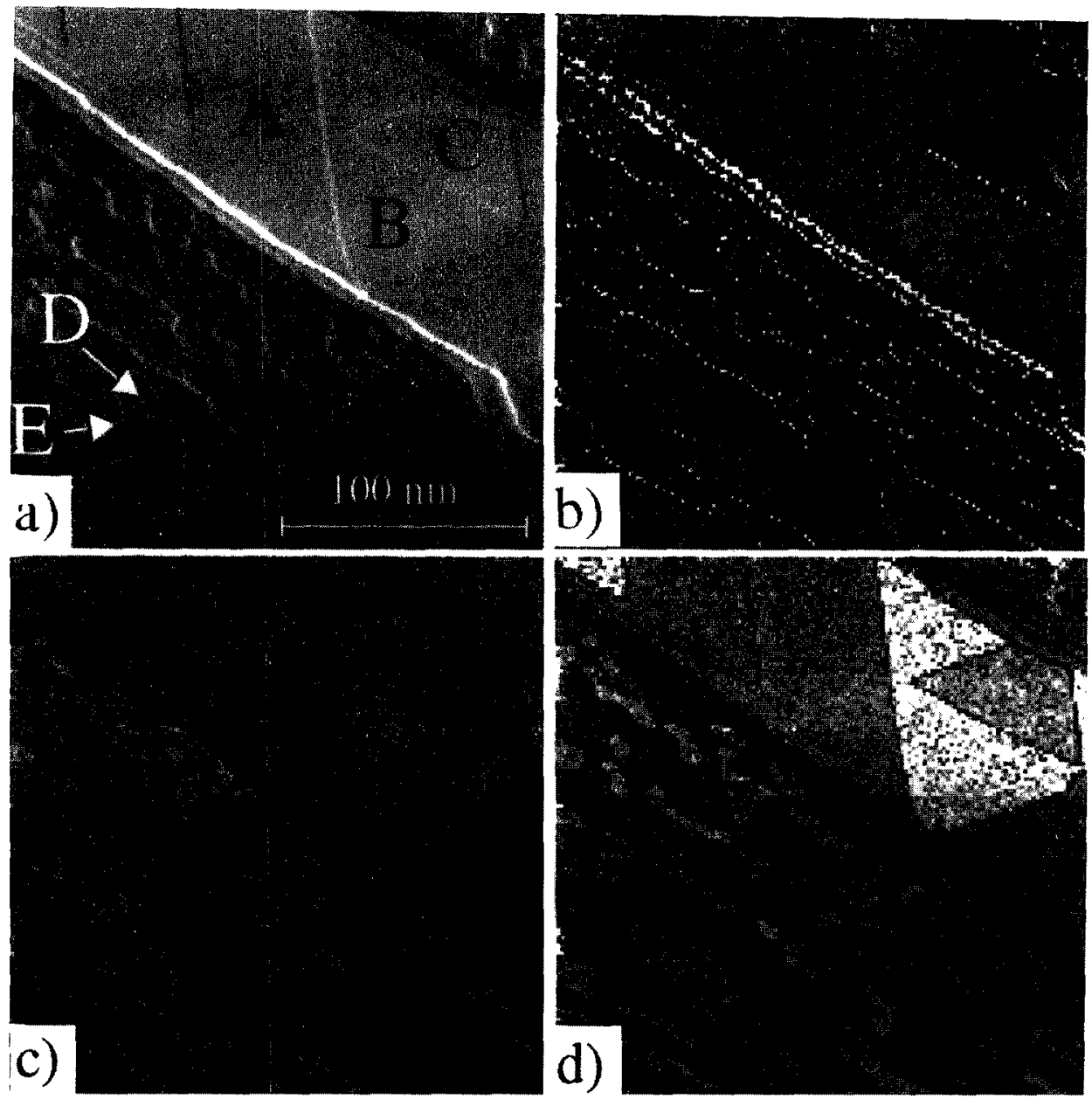

Fig. 3. (a) Constant-current STM topograph of $0.5 \mathrm{ML}$ Gd evaporated at $300 \mathrm{~K}$ on top of a sample surface similar to that shown in Fig. 1. On top of the high $\mathrm{Gd}(0001)$ island $(A)$ triangular shaped $\mathrm{Gd}$ island (e.g. $B$ ) appear. Maps of the differential conductivity $\mathrm{d} I / \mathrm{d} U$ measured at different sample bias are shown in (b) $U=+0.4 \mathrm{~V}$, (c) $U=-0.15 \mathrm{~V}$, and (d) $U=-0.2 \mathrm{~V}$.

at approximately $U=-0.1 \mathrm{~V}$. Further tuning the sample bias towards negative values caused a stronger rise of the differential conductivity above the island than above the monolayer.

We attribute the peaks at $U=+0.3 \mathrm{~V}$ and $U=-0.1 \mathrm{~V}$ measured above the $\mathrm{Gd}(0001)$ island to the empty and occupied part of the exchange split surface state, respectively. The exchange splitting amounts $\approx 400 \mathrm{meV}$ at $293 \mathrm{~K}$. As characteristic far a surface state we found a strong sensitivity of these two electronic features towards adsorbates like hydrogen, oxygen, and carbon monoxide. These experimental results will be published in detail elsewhere. In contrast, the peak at 
$U=+0.3 \mathrm{~V}$ in the spectrum of the monolayer does not change up to an exposure of $5 \mathrm{~L}$ and does therefore not represent a surface state.

Until now we have shown that the surface state exists above $\mathrm{Gd}(0001) /$ $\mathrm{W}(110)$ for $22 \mathrm{ML} \leq \Theta_{\text {loc }} \leq 4 \mathrm{ML}$. Its exchange splitting does not depend on the local coverage at $293 \mathrm{~K}$. Furthermore, the first ML of $\mathrm{Gd} / \mathrm{W}(110)$ exhibits a peak in the $\mathrm{d} I / \mathrm{d} U$-signal which does not represent a surface state. The question arises at which minimal film thickness the surface state appears above the Gd(0001) surface? To unravel this question we evaporated 0.5 ML Gd on a sample surface similar to Fig. 1a at $300 \mathrm{~K}$. As shown in Fig. 3a this leads to homoepitaxial growth of $\mathrm{Gd} / \mathrm{Gd}(0001) / \mathrm{W}(110)$ (e.g. the triangular shaped islands named $B$ and $C$ on top of the large island $A$ ) and to nucleation of second $(D)$ and third layer patches $(E)$ on top of the closed first monolayer. Again, the differential conductivity was measured simultaneously with the topography. Figures $3 \mathrm{~b}-\mathrm{d}$ show maps of the $\mathrm{d} I / \mathrm{d} U$-signal at three particular bias voltages: (b) $U=+0.4 \mathrm{~V}$, (c) $U=-0.15 \mathrm{~V}$, and (d) $U=-0.2 \mathrm{~V}$. Surprisingly, the monolayer high island $B$ exhibits a $\mathrm{d} I / \mathrm{d} U$-signal which differs from the underlying island $A$ best visible in Figs. 3c and 3d. Approximately $5-10 \%$ of the total number of islands were found with this property. In contrast, island $C$ which is grown on island $B$ does not deviate significantly from the $\mathrm{d} I / \mathrm{d} U$-signal of island $A$. One possible explanation for this change of the electronic structure with respect to the underlying (0001)-surface and most of the Gd islands of monolayer height is a stacking fault between the surface and the first subsurface layer.

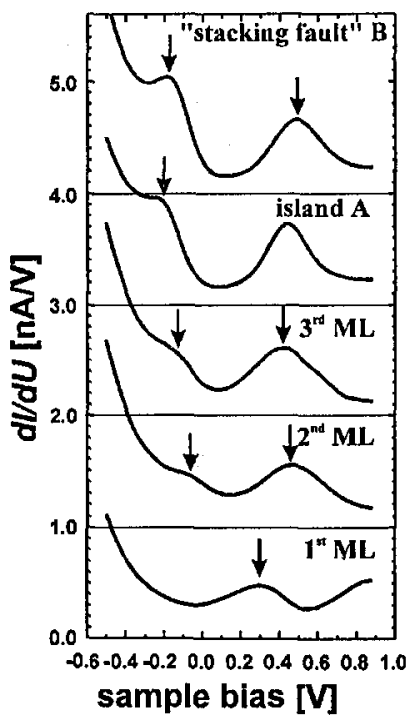

Fig. 4. Tunneling spectra measured at $T=117 \mathrm{~K}$ for local coverages $\Theta_{\mathrm{loc}}=1,2,3$, and $\geq 4 \mathrm{ML}$ ("island"). For local coverages of 2 and $3 \mathrm{ML}$ the surface state exists but the exchange splitting between is reduced. The spectrum of island $B$ in Fig. 3a is shifted by $\approx 20-30 \mathrm{meV}$, possibly due to a stacking fault. 
Furthermore, it is obvious from Fig. 3c that the electronic structure of second and third monolayer patches differs from each other (note the sites marked by arrows $D$ and $E$ in Fig. 3a). In Fig. 4 averaged spectra measured above Gd-patches/films with $\Theta_{l o c}=1,2,3$, and $\geq 4 \mathrm{ML}$, i.e. islands, are shown. Additionally, the spectrum of island $B$ which was supposed to exhibit a stacking fault is plotted. Again, one asymmetric peak measured above the Gd monolayer can be recognized. Already above double Gd layer patches $(D)$ the double peak structure as characteristic for the $\mathrm{Gd}(0001)$ surface state appears. By going to higher local coverages the exchange splitting increases up to the 4th layer. Since this measurement was performed at reduced temperature $(T=117 \mathrm{~K})$ the exchange splitting exceeds the value extracted from Fig. 2 . It should be mentioned in this context that the spectrum of island $B$ ("stacking fault") shows the same exchange splitting as island $A$. The contrast as visible in the maps of the differential conductivity originates from a shift of all electronic features by approximately $20-30 \mathrm{meV}$ towards higher binding energy.

\section{Summary}

We have investigated the dependence of the electronic properties of $\mathrm{Gd}(0001) / \mathrm{W}(110)$ for coverages which exceed one atomic monolayer. We found that the surface state exists already above the second ML. The exchange splitting increases by going from the second up to the fourth $\mathrm{ML}$ and remains constant for higher coverages. Occasionally, homoepitaxial growth of Gd/Gd(0001)/W(110) leads to a shift of the features in the $\mathrm{d} I / \mathrm{d} U$-spectra which may be caused by a stacking fault between the surface and the first subsurface layer.

\section{References}

[1] G. Binnig, H. Rohrer, Helv. Phys. Acta 55, 726 (1982).

[2] A. Baratoff, G. Binnig, H. Rohrer, J. Vac. Sci. Techn. B 1, 703 (1983).

[3] A. Selloni, P. Carnevali, E. Tosatti, C.D. Chen, Phys. Rev. B 31, 2602 (1985).

[4] R. Wiesendanger, M. Bode, R. Pascal, W. Allers, U.D. Schwarz, J. Vac. Sci. Technol. A 14, 1161 (1996).

[5] M. Bode, R. Pascal, M. Dreyer, R. Wiesendanger, Phys. Rev. B 54, R8385 (1996).

[6] R. Pascal, C. Zarnitz, M. Bode, R. Wiesendanger, Surf. Sci. Lett. 385, L990 (1997).

[7] R. Pascal, C. Zarnitz, M. Bode, R. Wiesendanger, Phys. Rev. B 56, 3636 (1997).

[8] D. Li, C.W. Hutchigs, P.A. Dowben, C. Hwang, R.T. Wu, M. Onellion, A.B. Andrews, J.L. Erskine, J. Magn. Magn. Mater. 99, 85 (1991).

[9] D. Li, P.A. Dowben, J.E. Ortega, F.J. Himpsel, Phys. Rev. B 49, 7734 (1994).

[10] D. Weller, S.F. Alvarado, W. Gudat, K. Schröder, M. Campagna, Phys. Rev. Lett. 54, 1555 (1985).

[11] H. Tang, D. Weller, T.G. Walker, J.C. Scott, C. Chappert, H. Hopster, A.W. Pang, D.S. Dessau, D.P. Pappas, Phys. Rev. Lett. 71, 444 (1993).

[12] E. Weschke, C. Schüssler-Langeheine, R. Meier, A.F. Fedorov, K. Starke, F. Hübinger, G. Kaindl, Phys. Rev. Lett. 77, 3415 (1996). 
[13] M. Farle, K. Baberschke, U. Stetter, A. Aspelmeier, F. Gebhardter, Phys. Rev. B. 47, 11571 (1993).

[14] M. Bode, R. Pascal, R. Wiesendanger, Surf. Sci. 344, 185 (1995).

[15] A. Aspelmeier, F. Gebhardter, K. Baberschke, J. Magn. Magn. Mater. 132, 22 (1994).

[16] J. Kołaczkiewicz, E. Bauer, Surf. Sci. 175, 487 (1986).

[17] E.D. Tober, R.X. Ynzunza, C. Westphal, C.S. Fadley, Phys. Rev. B 53, 5444 (1996).

[18] R. Pascal, Ch. Zarnitz, M. Bode, M. Getzlaff, R. Wiesendanger, Appl. Phys. A 65, 603 (1997). 